\title{
ANÁLISIS DE LOS NIVELES DE ACOSO ENTRE IGUALES Y SU RELACIÓN CON EL NIVEL DE ACTIVIDAD FÍSICA EN EDUCACIÓN PRIMARIA
}

\author{
Antonio Serrano Díaz ${ }^{1}$, Bernardino J. Sánchez-Alcaraz Martínez ${ }^{1}$, Javier Courel- \\ Ibáñez $^{1}$, Alberto Gómez-Mármol ${ }^{2}$ y Alfonso Valero-Valenzuela ${ }^{1}$ \\ ${ }^{1}$ Facultad de Ciencias del Deporte. Universidad de Murcia. \\ ${ }^{2}$ Facultad de Educación. Universidad de Murcia.
}

bjavier.sanchez@um.es

Fecha de recepción: julio 2018

Fecha de aceptación: octubre 2018

DOI: http://doi.org/10.15366/citius2018.11.2.005

\section{Resumen:}

Este estudio pretende conocer la relación entre la percepción de acoso entre iguales y el nivel de actividad física en estudiantes de Primaria. La muestra estuvo compuesta por 95 alumnos, con edades comprendidas entre los 9 y los 12 años, a los cuales se les administró el Cuestionario de Acoso entre Iguales y la traducción al español del Physician-based Assessment and Counseling for Exercise. Los resultados obtenidos mostraron unos niveles bajos tanto de actividad física como de percepción de acoso entre iguales. Se encontraron valores más altos de inconformismo de género y nivel de actividad física en los chicos, mientras que las chicas mostraron valores superiores en el maltrato verbal por falta de atractivo físico. Las comparaciones de nivel educativo muestran mayores niveles tanto en las conductas de acoso como en el nivel de actividad física en los alumnos de mayor edad. No se han encontrado correlaciones significativas entre el nivel de actividad física y las conductas de acoso.

Palabras clave: Acoso escolar, Deporte, Adolescentes, Educación.

Title: ANALYSIS OF BULLYING LEVELS AND ITS RELATIONSHIP WITH PHYSICAL ACTIVITY IN PRIMARY EDUCATION

Abstract:

The objective of this study is to understand the relationship between the perception of peer harassment and the level of physical activity in primary school students. The sample consisted of 95 students, aged between 9 and 12 years, who answered the Peer Harassment Questionnaire and the translation to Spanish of the Physician-based Assessment and Counselling for Exercise to measure both variables. The results obtained showed low levels of physical activity and perception of bullying among peers were obtained. Significant differences in gender nonconformity and higher level of physical activity were found in boys, while girls showed significantly higher values in verbal abuse due to lack of physical attractiveness. Comparisons of educational level show higher levels in all areas, both of harassment and physical activity level in older students. No significant correlations have been found between the level of physical activity and bullying behaviour, so it seems that at these ages may not yet have influence.

Keywords: Bullying, Sport, Adolescent, Education. 


\section{Introducción}

La violencia que se genera en las relaciones interpersonales ocasiona alta ansiedad y frustración, especialmente cuando se contempla en el contexto escolar, donde sus protagonistas son los escolares. Unas veces va dirigida hacia el profesorado, en otras ocasiones hacia la Institución y, en la mayoría de los casos, hacia los propios escolares. Esta problemática ya constituye una constante preocupación de los profesionales de la educación que precisa de un conocimiento de la situación mediante estrategias fiables y objetivas, que ayuden a prevenir sus efectos y controlar sus consecuencias, tanto en los directamente implicados como en el conjunto de la comunidad educativa (Cerezo, 2016).

La elevada incidencia del acoso entre iguales en las escuelas ha propiciado el incremento de los estudios dirigidos a analizar qué factores influyen en su desarrollo, principalmente en la etapa de la adolescencia en la que estas conductas resultan más graves y problemáticas (Cava, Musitu y Murgui 2006). La violencia del acoso entre iguales puede adoptar diferentes formas que se encuentran dentro de dos grandes campos: el bullying presencial y el no presencial. Dentro del presencial encontramos varias formas: físico, verbal o indirecto. El no presencial es debido a la gran extensión del uso de los dispositivos con conexión a internet entre la población estudiantil, encontrando una forma específica del bullying, -el ciberbullying- que utiliza estos medios para agredir (Cerezo, 2012; Garaigordobil y Oñederra, 2010).

La edad de los implicados es un dato de gran interés. Es cierto que la mayoría de los estudios sitúan la edad de mayor incidencia entre los 13 y 14 años, pero también es cierto que otros estudios confirman que esta edad cada vez es más temprana (8-9), lo que hace pensar que el fenómeno se encuentra en todas las edades escolares (Cerezo, Méndez y Ato, 2013). Aunque exista un blanco directo sobre el que el agresor dirige sus ataques, indudablemente el acoso entre iguales afecta a todos los participantes, víctimas, agresores/as y testigos (Avilés 2006). Para la víctima es para quien puede tener consecuencias más nefastas, ya que puede desembocar en fracaso y dificultades escolares, niveles altos y continuos de ansiedad y, más concretamente, ansiedad anticipatoria, insatisfacción, fobia a ir al colegio, riesgos físicos, y en definitiva conformación de una personalidad insegura e insana para el desarrollo correcto e integral de la persona.

Existen programas e iniciativas dirigidas a familias, docentes y discentes, con el fin básico de dar a conocer este fenómeno, sus formas más frecuentes o protocolos de actuación, con el objetivo de reducir y poner solución a este tipo de conflictos (Ortega, 2010). No obstante, una de las medidas de mayor interés y que más eficacia ofrecen son las referidas a la prevención (Serrano, 2006) y, más específicamente, las relacionadas con la práctica deportiva como una herramienta que permite aprender conductas sociales (Pelegrín, Martínez y Garcés de los Fayos, 2000). Del siguiente modo, el área de Educación Física se presenta como un contexto en el que desarrollar múltiples objetivos (Pérez-Soto y García-Canto, 2013), siendo la convivencia escolar uno de ellos (Sánchez-Alcaraz, López-Jaime, Valero-Valenzuela y Gómez-Mármol, 2017; Sánchez-Alcaraz, Mengual-García, Gómez-Mármol y Valero-Valenzuela, 2017). Sin embargo, son todavía escasos los estudios que han evaluado la relación entre los niveles de conductas de acoso entre iguales y la práctica de actividad física.

Por lo tanto, este estudio tiene como objetivo comprobar si el grado de actividad física en edad escolar podría ser un factor preventivo contra el bullying, analizando la relación que existe entre ambos, en el tercer ciclo de Educación Primaria y segundo de Educación Secundaria. Asimismo, se desarrollan propuestas para la prevención y solución de estas situaciones de violencia escolar mediante la práctica deportiva. 


\section{Método}

\subsection{Participantes}

La muestra estuvo compuesta por 96 estudiantes, con edades comprendidas entre los 9 y los 12 años, correspondientes a los cursos de $4^{\circ}$ de Educación Primaria (45 alumnos) y $6^{\circ}$ de Educación Primaria (50 alumnos), de los cuales 45 fueron chicos y 50 fueron chicas. Los participantes pertenecían a un Centro Educativo de la Región de Murcia.

\subsection{Instrumentos}

Acoso entre iguales: Se utilizó el Cuestionario de Acoso entre Iguales (CAI) diseñado y validado por Magaz (2012) (Anexo 1). Este instrumento evalúa dos escalas del acoso entre iguales: Conductas de Acoso (CAI-CA) y Conductas de Acoso de Género (CAI-CAG). La CAICA consta de 39 ítems, los cuales hacen referencia a distintas conductas de acoso que los niños pueden sufrir por parte de otros chicos/as. A partir de esta escala, pueden evaluarse de forma separada las siguientes formas de acoso entre iguales (subescalas): (1) maltrato verbal (11 ítems; p.ej. «me insultan»), (2) exclusión social directa (5 ítems; p.ej. «no se quieren sentar conmigo»), (3) amenazas (4 ítems; p.ej. «me amenazan para que haga cosas que no quiero»), (4) ciberbullying (4 ítems; p.ej. «cuando chateo con otros niños, se meten conmigo»), (5) exclusión social indirecta (4 ítems; p.ej. «me dejan de hablar»), (6) agresión basada en objetos (3 ítems; p.ej. «me tiran cosas»), y (7) maltrato físico ( 8 ítems; p.ej. «me pegan patadas»). La CAI-CAG está formada por 10 ítems, 5 para chicos y 5 para chicas. El contenido de los ítems específicos para los chicos hace referencia a dos subescalas: (1) ser objeto de maltrato verbal por falta de atractivo físico ( 2 ítems; p.ej. «dicen a otros que soy feo») y (2) inconformismo de género (3 ítems; p.ej., «me llaman nenaza»). En el caso de las chicas, el contenido de los ítems se corresponde a dos subescalas: (1) ser objeto de maltrato verbal por falta de atractivo físico (2 ítems; p.ej. «dicen a otros que soy fea») y (2) inconformismo de género (3 ítems; p.ej., «me llaman marimacho»). El cuestionario es evaluado en una escala tipo Likert de tres opciones, desde 0 («Nunca») hasta 2 («Muchas veces»).

Nivel de actividad física: Para evaluar el nivel de actividad física se utilizó el Physicianbased Assessment and Counseling for Exercise (PACE) diseñado por Proschaska, Sallis y Long (2001). Este instrumento comprende dos preguntas sobre la frecuencia de práctica de actividad física durante al menos 60 minutos en la última semana y en una semana normal y consta de la siguiente información introductoria: «Actividad física es cualquier actividad que incrementa tu ritmo cardiaco y hace que se acelere tu respiración. Actividad física se puede realizar haciendo deporte, jugando con amigos o caminando al colegio. Algunos ejemplos de actividad física son correr, caminar de forma vigorosa, montar en patines o monopatín, bailar, nadar, fútbol, baloncesto, voleibol, balonmano. No incluyas el tiempo en las clases de educación física escolar». Para clasificar a un sujeto como activo se considera que la media de estas dos respuestas debe ser $\geq 5$ días (Martínez-Gómez et al., 2009).

\subsection{Procedimiento}

El diseño de este estudio corresponde a una investigación empírica con metodología cuantitativa, concretamente un estudio descriptivo de poblaciones mediante encuestas con muestras probabilísticas de tipo transversal (Thomas y Nelson, 2007). El artículo fue aprobado por la Comisión de Ética de la Universidad de Murcia. Posteriormente, se solicitó el consentimiento de las familias y del Centro Educativo. Finalmente, los estudiantes cumplimentaron en horario escolar, los diferentes cuestionarios del estudio. Durante la aplicación, al menos un investigador estaba presente en el aula, que garantizó el anonimato de las respuestas. Los participantes contestaron los cuestionarios en 25 minutos aproximadamente, sin que ninguno de los ellos informara de problemas en la cumplimentación de los mismos. 


\subsection{Análisis de datos}

En primer lugar, se calcularon los estadísticos descriptivos (frecuencias, medias y desviaciones típicas) de las variables de acoso entre iguales y nivel de actividad física, tanto para el total de la muestra como atendiendo a las categorías de las variables independientes (sexo y edad). Posteriormente, se realizó la prueba de Kolmogorov-Smirnov para comprobar la normalidad de la muestra y se analizaron las diferencias significativas entre los grupos de sexo y edad mediante la prueba no paramétrica U de Mann Whitney. Se consideró una significación al 95\%. Los resultados fueron analizados mediante el paquete estadístico SPSS 21.0 para Macintosh.

\section{Resultados}

En la tabla 1 se muestran los resultados descriptivos de la percepción de acoso entre iguales en las siete dimensiones en las que se agrupan los ítems de Conductas de Acoso y las cuatro dimensiones de Conductas de Acoso de Género, ambos dentro del Cuestionario de Acoso entre Iguales. También se muestran los resultados del nivel de actividad física, correspondientes al cuestionario Physician-based Assessment and Counseling for Exercise. En líneas generales, los resultados obtenidos para toda la muestra revelan unos índices bajos en la percepción de acoso entre iguales y un índice medio-bajo en nivel de actividad física. En conductas de acoso se observa unos niveles superiores de percepción de acoso entre iguales en la dimensión maltrato verbal y unos niveles inferiores en la dimensión ciberbullying. Por lo que respecta a conductas de acoso por género, los chicos tienen niveles superiores en la dimensión de género y niveles inferiores en la dimensión de atractivo físico con respecto a las chicas.

Tabla 1

Resultados descriptivos del nivel de actividad física y la percepción de acoso entre iguales para el total de la muestra.

\begin{tabular}{|c|c|c|}
\hline & Media & $\begin{array}{l}\text { Desviación } \\
\text { típica }\end{array}$ \\
\hline \multicolumn{3}{|l|}{ Conductas de Acoso } \\
\hline Maltrato verbal & .38 & .38 \\
\hline Maltrato físico & .24 & .31 \\
\hline Exclusión social directa & .24 & .40 \\
\hline Amenazas & .15 & .31 \\
\hline Ciberbullying & .04 & .15 \\
\hline Exclusión social indirecta & .27 & .41 \\
\hline Agresión basada objetos & .14 & .25 \\
\hline \multicolumn{3}{|l|}{ Conductas de Acoso de Género } \\
\hline Inconformismo género (chicos) & .25 & .47 \\
\hline $\begin{array}{l}\text { Maltrato verbal por falta de atractivo } \\
\text { físico (chicos) }\end{array}$ & .13 & .28 \\
\hline Inconformismo género (chicas) & .19 & .35 \\
\hline $\begin{array}{l}\text { Maltrato verbal por falta de atractivo } \\
\text { físico (chicas) }\end{array}$ & .19 & .40 \\
\hline Nivel actividad física & 2.77 & 1.69 \\
\hline
\end{tabular}

Nota: Des. típ. $=$ Desviación típica. 
Por otro lado, la tabla 2 recoge datos de la percepción de acoso entre iguales y nivel de actividad física en función del género de la muestra. De forma general, en los resultados obtenidos en los chicos, se observa los niveles más altos en la dimensión de maltrato verbal y la dimensión con niveles más bajos es ciberbullying, ambas dimensiones se encuentran dentro de conductas de acoso. En cuanto a las chicas, los niveles más altos y más bajos coinciden en la misma dimensión que en chicos, si bien los resultados muestran un índice inferior en ambas dimensiones con respecto al género masculino. Por otra parte, en las dimensiones de conductas de acoso, los chicos mostraron un mayor índice en todas las dimensiones a excepción de exclusión social directa y exclusión social indirecta, donde las chicas fueron ínfimamente superiores, sin haber significación entre ambos. En las dimensiones de conductas de acoso por género, las chicas tienen un índice mayor en la dimensión atractivo físico que los chicos. En cuanto al nivel de actividad física comparada por género, se aprecia una significación de 0.028 , con una media cercana a un punto de diferencia de chicos a chicas.

Tabla 2

Diferencias en nivel de actividad física y la percepción de acoso entre iguales en función del género

\begin{tabular}{lccccc}
\hline & \multicolumn{2}{c}{ Masculino } & \multicolumn{2}{c}{ Femenino } & \\
& Media & Desv. típ. & Media & Desv. típ. & Sig. \\
\hline Conductas de Acoso & & & & & \\
$\quad$ Maltrato verbal & .43 & .45 & .34 & .31 & .591 \\
$\quad$ Maltrato físico & .28 & .32 & .21 & .30 & .174 \\
$\quad$ Exclusión social directa & .23 & .44 & .24 & .36 & .313 \\
$\quad$ Amenazas & .21 & .39 & .10 & .22 & .194 \\
$\quad$ Ciberbullying & .06 & .18 & .03 & .10 & .254 \\
$\quad$ Exclusión social indirecta & .26 & .38 & .28 & .44 & .696 \\
$\quad$ Agresión basada objetos & .15 & .23 & .14 & .27 & .358 \\
Conductas de Acoso de Género & .25 & .47 & .19 & .35 & $.000^{* *}$ \\
$\quad$ Inconformismo género & .13 & .28 & .19 & .40 & $.000^{* *}$ \\
$\quad$ Maltrato verbal por falta de & .13 & & & & \\
$\quad$ atractivo físico & 3.20 & 1.69 & 2.39 & 1.60 & $.028^{*}$ \\
Nivel actividad física & & & & & \\
\hline
\end{tabular}

Nota: $* \mathrm{p}<.05 ; * * \mathrm{p}<.01$ 
Los datos recogidos de las diferencias en nivel de actividad física y la percepción de acoso entre iguales en función del nivel educativo se observan en la tabla 3. En todas las dimensiones de conductas de acoso se observa unos niveles superiores de los alumnos de sexto con respecto a los alumnos de cuarto, donde la dimensión de maltrato verbal es la que tiene mayor índice y el ciberbullying la dimensión de menor índice de percepción de acoso entre iguales en ambos niveles educativos. Se aprecian dos dimensiones con significación, una es ciberbullying (0.016) y la otra es agresión basada en objetos (0.047). De igual manera, en las dimensiones de conductas de acoso por género se aprecia un mayor índice en el nivel educativo de sexto sobre cuarto, a excepción del atractivo físico masculino. Existe una significación entre ambos niveles en la dimensión de género masculino (0.031) y también en lo que respecta al nivel de actividad física (0.003).

Tabla 3

Diferencias en nivel de actividad física y la percepción de acoso entre iguales en función del nivel educativo.

$4^{\circ}$ PRIMARIA $\quad 6^{\circ}$ PRIMARIA

\begin{tabular}{lccccc} 
& Media & Desv. típ. & Media & Desv. típ. & Sig. \\
\hline Conductas de Acoso & .30 & .27 & .47 & .47 & .183 \\
$\quad$ Maltrato verbal & .30 & .27 & .47 & .47 & .183 \\
$\quad$ Maltrato físico & .18 & .19 & .31 & .39 & .329 \\
$\quad$ Exclusión social directa & .17 & .23 & .31 & .51 & .507 \\
$\quad$ Amenazas & .08 & .15 & .23 & .41 & .156 \\
$\quad$ Ciberbullying & .01 & .07 & .08 & .19 & $.016^{*}$ \\
$\quad$ Exclusión social indirecta & .23 & .44 & .31 & .37 & .111 \\
$\quad$ Agresión basada objetos & .09 & .19 & .20 & .30 & $.047^{*}$ \\
Conductas de Acoso de Género & & & & & \\
$\quad$ Inconformismo género (chicos) & .12 & .29 & .42 & .59 & $.031^{*}$ \\
$\quad$ Maltrato verbal por falta de & .08 & .22 & .20 & .34 & .220 \\
$\quad$ atractivo físico (chicos) & .20 & .27 & .18 & .43 & .290 \\
$\quad$ Inconformismo género (chicas) & .16 & .23 & .22 & .52 & .518 \\
$\quad \begin{array}{l}\text { Maltrato verbal por falta de } \\
\quad \text { atractivo físico (chicas) }\end{array}$ & 2.30 & 1.71 & 3.30 & 1.52 & $.003^{*}$ \\
\hline Nivel actividad física & & & & & \\
\hline
\end{tabular}

Nota: $* \mathrm{p}<.05 ; * * \mathrm{p}<.01$ 
Por último, se analizaron las correlaciones entre las diferentes variables del acoso entre iguales y el nivel de actividad física. En este sentido, no se encontraron relaciones significativas entre el nivel de actividad física y ninguna de las dimensiones del acoso entre iguales.

Tabla 4

Correlaciones entre nivel de actividad fisica y la percepción de acoso entre iguales

\begin{tabular}{|c|c|c|}
\hline & & $\begin{array}{c}\text { Nivel de Actividad } \\
\text { Física }\end{array}$ \\
\hline \multirow{2}{*}{ Maltrato Verbal } & $\begin{array}{l}\text { Coef. de } \\
\text { correlación }\end{array}$ & -.046 \\
\hline & Sig. (Bilateral) & .656 \\
\hline \multirow{2}{*}{ Maltrato Físico } & $\begin{array}{l}\text { Coef. de } \\
\text { correlación }\end{array}$ & .010 \\
\hline & Sig. (Bilateral) & .922 \\
\hline \multirow{2}{*}{ Exclusión Social Directa } & $\begin{array}{l}\text { Coef. de } \\
\text { correlación }\end{array}$ & -.129 \\
\hline & Sig. (Bilateral) & .213 \\
\hline \multirow{2}{*}{ Amenazas } & $\begin{array}{l}\text { Coef. de } \\
\text { correlación }\end{array}$ & -.052 \\
\hline & Sig. (Bilateral) & .620 \\
\hline \multirow{2}{*}{ Ciberbullying } & $\begin{array}{l}\text { Coef. de } \\
\text { correlación }\end{array}$ & -056 \\
\hline & Sig. (Bilateral) & .588 \\
\hline \multirow{2}{*}{ Exclusión Social Indirecta } & $\begin{array}{l}\text { Coef. de } \\
\text { correlación }\end{array}$ & -.137 \\
\hline & Sig. (Bilateral) & .187 \\
\hline \multirow{2}{*}{ Agresión Basada Objeto } & $\begin{array}{l}\text { Coef. de } \\
\text { correlación }\end{array}$ & -.045 \\
\hline & Sig. (Bilateral) & .665 \\
\hline \multirow{2}{*}{$\begin{array}{l}\text { Inconformismo género } \\
\text { Masculino }\end{array}$} & $\begin{array}{l}\text { Coef. de } \\
\text { correlación }\end{array}$ & -.165 \\
\hline & Sig. (Bilateral) & .280 \\
\hline \multirow{2}{*}{$\begin{array}{l}\text { Maltrato verbal por falta de } \\
\text { atractivo físico Masculino }\end{array}$} & $\begin{array}{l}\text { Coef. de } \\
\text { correlación }\end{array}$ & -.195 \\
\hline & Sig. (Bilateral) & .198 \\
\hline \multirow{2}{*}{$\begin{array}{l}\text { Inconformismo género } \\
\text { Femenino }\end{array}$} & $\begin{array}{l}\text { Coef. de } \\
\text { correlación }\end{array}$ & .062 \\
\hline & Sig. (Bilateral) & .671 \\
\hline \multirow{2}{*}{$\begin{array}{l}\text { Maltrato verbal por falta de } \\
\text { atractivo físico Femenino }\end{array}$} & $\begin{array}{l}\text { Coef. de } \\
\text { correlación }\end{array}$ & .088 \\
\hline & Sig. (Bilateral) & .543 \\
\hline
\end{tabular}




\section{Discusión}

El objetivo de este trabajo fue conocer la relación entre el nivel de actividad física y la percepción de acoso entre iguales (bullying) a nivel escolar de Educación Primaria en un centro de la Región de Murcia. A nivel general, los resultados mostraron unos niveles bajos en la percepción de conductas de acoso entre iguales, destacando el maltrato verbal como la dimensión con niveles más altos y el ciberbullying como la dimensión más baja de conductas de acoso. Estos resultados coinciden con los estudios de Garaigordobil y Oñederra (2008), que evaluaron las conductas de acoso en 5.983 estudiantes de 169 centros educativos de la Comunidad Autónoma del País Vasco, donde la gran mayoría de los escolares se sienten bien tratados por sus compañeros, con un índice de bullying obtenido del 5,8\% de víctimas en Primaria.

Por otro lado, el nivel de actividad física de los estudiantes es bajo, con valores similares a los estudios de Luengo (2007), en los que existe un porcentaje muy bajo de alumnos que realiza actividades extraescolares, entre 3 y 4 veces por semana (7.8\%) y Martínez, Contreras, Aznar y Lera (2012), en el cual solamente un 22,2\% de los niños/as cumplían las recomendaciones internacionales de actividad física saludable (mínimo 1 hora de actividad vigorosa al día). Estos resultados pueden vincularse con otros datos preocupantes reflejados en el estudio más reciente en la población pediátrica (Ortega et al., 2015), utilizando los criterios de la Organización Mundial de la Salud (OMS), que han situado a España en el segundo país con mayor obesidad infantil en Europa y cuyas causas principales son el sedentarismo y la mala alimentación. Por lo que respecta a los niveles bajos de actividad física, estudios con una línea de investigación sobre el nivel de actividad física de escolares, muestran un aumento de hábitos sedentarios en los últimos años en España, como, por ejemplo, Cuberos et al. (2015), donde 3 de cada 10 escolares no realizaban actividad física cotidiana. Este porcentaje se asemeja al mostrado por De Hoyo y Sañudo (2007), cuyos datos reflejaron un $25 \%$ de inactividad. De manera análoga, el estudio de Casado et al. (2009), presentó un 18\% de inactividad.

En función del género, se observó que los chicos percibían más acoso entre iguales que las chicas en casi la totalidad de las dimensiones, a excepción de exclusión social directa e indirecta, donde los niveles de las chicas eran levemente superiores. De este modo, son varios los autores que han afirmado que, a estas edades, los chicos realizan y sufren más acoso que las chicas, lo que puede deberse a que muchos niños no consideren el acoso verbal o relacional como un tipo de bullying (Postigo, González, Mateu, Ferrero y Martorell, 2009). Por otro lado, se encontraron diferencias significativas en las conductas de acoso de género, en las que se encontraron niveles superiores de inconformismo de género en los chicos y de maltrato verbal por falta de atractivo físico en las chicas. Una de las principales causas se encuentra en el impacto que tiene en las adolescentes el modelo estético corporal vigente en la cultura occidental. En esta línea, son varios los estudios que han mostrado unos niveles superiores de insatisfacción con la imagen corporal en el género femenino (Sáez y Mateo, 2002), lo que podría relacionarse con unos niveles más altos de acoso en esta dimensión. Además, las mujeres que se aproximan más al modelo estético delgado se sienten más atractivas, mejor físicamente y con un autoconcepto más alto (Fernández-Bustos et al., 2015).

Finalmente, con respecto a los niveles de actividad física, destacan unos valores significativamente superiores en los chicos frente a las chicas. Estos datos están en la línea de los estudios de Martínez y Veiga (2007) y Ángel, García y Zamorano (2007), que afirman que, en primaria, los niños realizan más actividad física y deporte, tanto federado como no federado. Siguiendo a Casado et al. (2009), estas diferencias pueden ser debidas a varios factores, pero principalmente a que los padres suelen incitar más a los niños al deporte desde pequeños que a las niñas, o que los juegos deportivos más populares suelen estar más asociados al tipo de juegos que les gusta a los niños y no a las niñas. 
Atendiendo a los resultados en función del nivel educativo, los datos mostraron valores significativamente superiores en los alumnos de $6^{\circ}$ de Primaria en las dimensiones de ciberbullying y agresión basada en objetos. Además, en el género masculino, se encontró un nivel significativamente superior de inconformismo de género en los alumnos de $6^{\circ}$ de Primaria. Estos niveles superiores de conductas de acoso en alumnos de mayor edad pueden deberse, tal y como afirman Cepeda-Cuervo, Pacheco-Durán, García-Barco y Piraquive-Peña, (2008) y Cerezo (2009), a una tendencia por la ampliación hacia los extremos, lo que evidencia que el fenómeno se va generalizando a todas las edades escolares (Cerezo, 2005). Además, se determinó unos niveles superiores en el nivel de actividad física en los alumnos de $6^{\circ}$ de Primaria. Estos datos siguen la línea de los estudios de Moral, Redecillas y Martínez (2012), que afirman que el nivel de actividad física se incrementa con la edad hasta la llegada de la adolescencia, en la que sufre un descenso.

Por último, no se han encontrado correlaciones significativas entre el nivel de actividad física y las conductas de acoso. De este modo, aunque son muchos los estudios que han mostrado unos niveles superiores en valores como la autoestima, el liderazgo o el respeto a los demás en aquellos estudiantes que practican más actividad física (Gómez-Mármol et al., 2017), no parece que a estas edades pueda tener aún influencia.

Además, el estudio presenta unas limitaciones que deben ser tomadas en cuenta a la hora de interpretar los resultados. En primer lugar, el número de alumnos encuestados es muy escaso para poder generalizar estos resultados, por lo que se hace necesario incrementar el tamaño muestral incluyendo, por ejemplo, estudiantes de Educación Secundaria y Bachillerato. Además, con respecto a la evaluación de los niveles de actividad física, no se han incluido variables como, por ejemplo, si la actividad es individual o colectiva, el tipo de actividad o deporte practicado, o si lo desarrollan o no a nivel federativo, variables que si han sido tomadas en cuenta en otros estudios similares (García, Gutiérrez, González y Valero, 2012; Li, Wright, Rukavina y Pickering, 2008). Finalmente, futuros trabajos podrían evaluar otros aspectos relacionados con las conductas de acoso entre iguales, como los escenarios en las que se producen, estrategias de afrontamiento, personajes implicados, y confidentes.

\section{Conclusiones}

1. Los estudiantes mostraron, en general, unos niveles bajos de nivel de actividad física y de percepción de conductas de acoso entre iguales. Además, la conducta de acoso más percibida fue el maltrato verbal y la menos percibida el ciberbullying.

2. Los chicos presentaron valores significativamente más altos que las chicas en las dimensiones de inconformismo de género y nivel de actividad física. Las chicas mostraron valores significativamente más altos que los chicos en la dimensión de maltrato verbal por falta de atractivo físico.

3. Los alumnos de $6^{\circ}$ de Primaria obtuvieron niveles significativamente superiores en las dimensiones de ciberbullying, agresión basada en objetos, inconformismo de género (chicos) y nivel de actividad física.

4. En el nivel de actividad física no se encontraron correlaciones significativas con ninguna de las dimensiones del estudio.

\section{Referencias bibliográficas}

Ángel, J., García, E., y Zamorano, M. (2007). Estereotipos de género, actividad física y escuela: La perspectiva del alumnado. Profesorado. Revista de Currículum y Formación de Profesorado, 11(2). 
Avilés, J. (2006). Bullying intimidación y maltrato entre el alumnado. Bilbao: STEE-EILAS.

Aznar, S. y Webster, T. (2006). Actividad física y salud en la infancia y adolescencia: guía para todas las personas que participan en su educación. Ministerio de Educación.

Casado, C., Alonso, N., Hernández, V. y Jiménez, R. (2009). Actividad física en niños españoles. Factores asociados y evolución 2003-2006. Revista Pediatría Atención Primaria, 11(42), 219-231.

Cava, M. J., Musitu, G., y Murgui, S. (2006). Familia y violencia escolar: el rol mediador de la autoestima y la actitud hacia la autoridad institucional. Psicothema, 18(3).

Cepeda-Cuervo, E., Pacheco-Durán, P. N., García-Barco, L., y Piraquive-Peña, C. J. (2008). Acoso escolar a estudiantes de educación básica y media. Revista de salud pública, 10(4), 517-528. https://doi.org/10.1590/S0124-00642008000400002

Cerezo F (2005). La violencia en la escuela. VII Reunión Internacional Biología y Sociología de la Violencia. Valencia.

Cerezo, F. (2009). Bullying: análisis de la situación en las aulas españolas. International Journal of Psychology and Psychological Therapy, 9(3).

Cerezo, F. (2012). Bullying a través de las TIC. Sapiens Research. Boletín Científico Sapiens Research, 2(2), 24-29.

Cerezo, F. (2016). El profesorado ante el bullyng. Reflexiones, instrumentos y estrategias para abordarlo. El aprendizaje de los valores a través del deporte. Consejo Escolar de la Región de Murcia, pp.40-53.

Cerezo, F., Méndez, I. y Ato, M. (2013). Moderating role of family and friends' factors between dissocial behavior and consumption in adolescents. International Journal of Clinical and Health Psychology, 13(3). 171-180. https://doi.org/10.1016/S1697$\underline{2600(13) 70021-8}$

Cuberos, R. C., Martínez, A. M., Sánchez, M. C., Garcés, T. E., Moral, P. Á. V., y Ortega, F. Z. (2015). Relación entre bullying, género y actividad física: estudio en escolares de la provincia de Granada. TRANCES. Transmisión del Conocimiento Educativo y de la Salud, (6), 791-810.

De Hoyo, M. y Sañudo, B. (2007). Motivos y hábitos de práctica de actividad física en escolares de 12 a 16 años en una población rural de Sevilla. Revista Internacional de Medicina y Ciencias de la Actividad Física y del Deporte, 7(26), 87-98.

Fernández-Bustos, J. G., González-Martí, I., Contreras, O., y Cuevas, R. (2015). Relación entre imagen corporal y autoconcepto físico en mujeres adolescentes. Revista Latinoamericana de Psicología,47(1), 25-33. https://doi.org/10.1016/S01200534(15)30003-0

Garaigordobil, M. y Oñederra, J. A. (2010). La violencia entre iguales. Madrid. Pirámide

Garaigordobil, M. y Oñederra, J. A. (2008). Bullying: Incidence of peer violence in the schools of the Autonomous Community of the Basque Country. International Journal of Psychology and Psychological Therapy, 8(1). 
García, L. M., Gutiérrez, D., González, S. y Valero, A. (2012). Cambios en la empatía, la asertividad y las relaciones sociales por la aplicación del modelo de instrucción educación deportiva. Revista de Psicología del Deporte, 21(2), 7-16.

Gómez-Mármol, A., Sánchez-Alcaraz, B.J., De la Cruz, E., Valero, A., González, S. (2017). Personal and social responsibility development through sport participation in youth scholars. Journal of Phyisical Education and Sport, 17(2), 775-782.

Li, W., Wright, P. M., Rukavina, P. y Pickering, M. (2008). Measuring students' perceptions of personal y social responsibility y its relationship to intrinsic motivation in urban physical education. Journal of Teaching in Physical Education, 27, 167-178. https://doi.org/10.1123/jtpe.27.2.167

Luengo, C. (2007). Actividad físico-deportiva extraescolar en alumnos de primaria. Revista Internacional de Medicina y Ciencias de la Actividad Física y del Deporte, 7(27), 174184.

Magaz, A. (2012). El maltrato entre iguales (bullying): Epidemiología y características psicológicas y sociodemográficas. Tesis doctoral. Universidad Nacional de Educación a Distancia (no publicada).

Martínez, D., y Veiga, O. L. (2007). Insatisfacción corporal en adolescentes: relaciones con la actividad física e índice de masa corporal. Revista Internacional de Medicina y Ciencias de la Actividad Física y del Deporte, 7(27), 253-265.

Martínez, J., Contreras, O. R., Aznar, S., y Lera, Á. (2012). Niveles de actividad física medido con acelerómetro en alumnos de $3^{\circ}$ ciclo de Educación Primaria: actividad física diaria y sesiones de Educación Física. Revista de Psicología del Deporte, 21(1), 117-123.

Martínez-Gómez, D., Martínez de Haro, V., Del Campo, J., Zapatera, B., Welk, G. J., Villagra, A. et al. (2009). Validez de cuatro cuestionarios para valorar la actividad física en adolescentes españoles. Gaceta Sanitaria, 23(6), 512-517. https://doi.org/10.1016/j.gaceta.2009.02.013

Moral, J. E., Redecillas, M. T., y Martínez, E. J. (2012). Hábitos sedentarios de los adolescentes andaluces. Journal of Sport \& Health Research, 4(1).

Ortega, R. (2010). Agresividad injustificada, bullying y violencia escolar. Alianza Editorial.

Ortega, R., López-Sobaler, A., Aparicio, A., González, L., Navia, B., y Pera, J.M. (2015). Estudio de Vigilancia del Crecimiento, Alimentación, Actividad Física, Desarrollo Infantil y Obesidad en España. Ministerio de Sanidad, Servicios Sociales e Igualdad.

Pelegrín, A., Martínez, F. y Garcés de los Fayos, E.J. (2000). La actividad física y el deporte como factores «controladores» de conductas antisociales y delictivas. II Congreso Iberoamericano de Psicología del Deporte, Huelva.

Pérez-Soto, J. J. y García-Canto, E. (2013). Competencias básicas y Educación Física en primaria: una aclaración conceptual. Trances, 5(3), 229-250.

Postigo, R. González, C. Mateu, J. Ferrero, C. y Martorell (2009) Diferencias conductuales según género en convivencia escolar. Psicothema, 21, 453-458.

Proschaska, J. J., Sallis, J. F. y Long, B. (2001). A physical activity screening measure for use with adolescents in primary care. Archives of Paedriatic and Adolescent Medicine, 155, 554-559. https://doi.org/10.1001/archpedi.155.5.554 
Sáez, M. S. C., y Mateo, C. M. (2002). Índice de masa corporal, preocupación por la delgadez y satisfacción corporal en adolescentes. Revista de psicología general y aplicada: Revista de la Federación Española de Asociaciones de Psicología, 55(3), 455-474.

Sánchez-Alcaraz, B.J., López-Jaime, G., Valero-Valenzuela, A. y Gómez-Mármol, A. (2017). Los programas de educación en valores a través de la educación física y el deporte. Actividad Física y Deporte, Ciencia y Profesión, 28, 45-58.

Sánchez-Alcaraz, B.J., Mengual-García, B., Gómez-Mármol, A., y Valero-Valenzuela, A. (2017). Observación de conductas que alteran la convivencia en las clases de educación física y relación con la violencia escolar cotidiana. Revista de Educación Física, 35(4), $1-8$.

Serrano, A. (2006). Acoso y violencia en la escuela: cómo detectar, prevenir y resolver el bullying. Madrid: Ariel.

Thomas, J. R., y Nelson, J. K. (2007). Métodos de investigación en actividad física. Barcelona: Paidotribo. 